\title{
Comprehensive and combined omics analysis reveals factors of ischemia-reperfusion injury in liver transplantation
}

\author{
Shanzhou Huang ${ }^{\ddagger 1,2,3}$, Weiqiang Ju ${ }^{\ddagger 1,2,3}$, Zebin Zhu ${ }^{\ddagger 1,2,3}$, Ming Han ${ }^{1,2,3}$, Chengjun \\ Sun 1,2,3, Yunhua Tang 1,2,3, Yuchen Hou ${ }^{1,2,3}$, Zhiheng Zhang 1,2,3, Jie Yang 1,2,3, Yixi \\ Zhang $^{1,2,3}$, Linhe Wang ${ }^{1,2,3}$, Fanxiong Lin'1,2,3, Haitian Chen 1,2,3, Rongxing Xie ${ }^{1,2,3}$, Caihui \\ Zhu $^{1,2,3}$, Dongping Wang ${ }^{1,2,3}$, Linwei Wu ${ }^{1,2,3}$, Qiang Zhao 1,2,3, Maogen Chen 1,2,3, Qi \\ Zhou*,4,5, Zhiyong Guo**,1,2,3 \& Xiaoshun He***,1,2,3 \\ ${ }^{1}$ Organ Transplant Center, The First Affiliated Hospital, Sun Yat-sen University, Guangzhou 510080, PR China \\ ${ }^{2}$ Guangdong Provincial Key Laboratory of Organ Donation \& Transplant Immunology, Guangzhou 510080, PR China \\ ${ }^{3}$ Guangdong Provincial International Cooperation Base of Science \& Technology (Organ Transplantation), Guangzhou 510080, PR \\ China \\ ${ }^{4}$ Department of General Surgery, Hui Ya Hospital of The First Affiliated Hospital, Sun Yat-sen University, Huizhou, Guangdong \\ 516081, PR China \\ ${ }^{5}$ Department of Liver Surgery, The First Affiliated Hospital of Sun Yat-Sen University, Guangzhou 510080, PR China \\ *Author for correspondence: hnzhouqi@163.com \\ **Author for correspondence: rockyucsf1981@126.com \\ ***Author for correspondence: gdtrc@163.com \\ ${ }^{\ddagger}$ Authors contributed equally
}

\begin{abstract}
Aim: To explore molecular mechanisms underlying liver ischemia-reperfusion injury (IRI). Materials \& methods: Four Gene Expression Omnibus datasets comprising liver transplantation data were collected for a comprehensive analysis. A proteomic analysis was performed and used for correlations analysis with transcriptomic. Results \& conclusion: Ten differentially expressed genes were co-upregulated in four Gene Expression Omnibus datasets, including ATF3, CCL4, DNAJB1, DUSP5, JUND, KLF6, NFKBIA, PLAUR, PPP1R15A and TNFAIP3. The combined analysis demonstrated ten coregulated genes/proteins, including $H B B, H B G 2$, CA1, SLC4A1, PLIN2, JUNB, HBA1, MMP9, SLC2A1 and PADI4. The coregulated differentially expressed genes and coregulated genes/proteins formed a tight interaction network and could serve as the core factors underlying IRI. Comprehensive and combined omics analyses revealed key factors underlying liver IRI, and thus having potential clinical significance.
\end{abstract}

First draft submitted: 3 November 2018; Accepted for publication: 2 January 2019; Published online: 31 January 2019

Keywords: combined analysis $\bullet$ comprehensive analysis $\bullet$ ischemia-reperfusion injury $\bullet$ liver transplantation $\bullet$ proteome $\bullet$ transcriptome

Tissue ischemia and reperfusion injury (IRI) contributes to the morbidity and mortality of a wide range of pathologies, including myocardial infarction, ischemic stroke, acute kidney injury, trauma and circulatory arrest [1]. IRI is also a major challenge during organ transplantation and cardiothoracic, vascular and general surgery and continues to be a vital source of morbidity among organ transplant recipients, especially among those receiving liver transplants [2]. With the enlarging shortage of available donor livers, the increased use of extended criteria for donor grafts further increases IRI, adversely affecting both the short-term and long-term outcomes of grafting and patient survival [3]. Numerous studies have investigated the benefits of pharmacological, heat shock and ischemic preconditioning interventions aimed at decreasing liver IRI [4], revealing limited benefits. Our center has made substantial efforts to conquer IRI, and we have developed a novel surgical technique called ischemia-free liver transplantation [5]. This technique constitutes an ultimate method to overcome IRI in liver transplantation, but its popularization still requires substantial effort. As a result, studying IRI and identifying the core genes and mechanisms underlying the process are of significant importance.

Future Medicine 
Comprehensive bioinformatics analysis has been increasingly used as an important method for studying various pathological and physiological conditions [6]. Performing comprehensive bioinformatics analysis by using multiple omics techniques by themselves or in combination can elucidate key factors that have potentially pathogenic impacts, such as gene expression, protein function and downstream pathways. With the rapid development of high-throughput sequencing technologies, several transcriptomic datasets comprising data on IRI during liver transplantation have become available in the Gene Expression Omnibus (GEO) database. Herein, we performed a comprehensive analysis of three GEO datasets as well as a GEO dataset constructed by our center. Moreover, we performed the first proteomic analysis of liver tissues to study liver IRI. Then, the transcriptome and proteome were used for combined analysis to reveal key factors in liver IRI.

\section{Materials \& methods}

\section{GEO datasets}

The GEO (www.ncbi.nlm.nih.gov/gds) is a public repository at the National Center of Biotechnology Information for storing high-throughput gene expression datasets. We selected potential GEO datasets according to the following inclusion criteria: organism: Homo sapiens; expression profiling by array; orthotopic liver transplantation; samples include preimplantation and postreperfusion liver tissues; and supported by a gene-cloud of biotechnology information (GCBI) analysis laboratory. Datasets with specimens from other organisms or expression profiling by RT-PCR (or genome variation profiling by small nucleotide polymorphism [SNP] array/SNP genotyping by SNP array) were excluded.

We used the search terms 'liver transplantation' MeSH Terms AND 'Homo sapiens' Organism AND 'Expression profiling by array' DataSet Type to identify potential GEO datasets. Then, we further screened these datasets according to the above inclusion criteria. Finally, three GEO datasets, GSE15480, GSE14951 and GSE12720, and the high-throughput gene expression sequencing dataset GSE113024 constructed by our center were included in our study. We extracted gene expression data from reperfused and preimplanted liver tissues to identify genes that were differentially expressed (DEGs) during IRI in these four chips. Coregulated DEGs were identified based on these top ranked DEGs.

\section{Transcriptome analysis}

The transcriptome profiles of CLT liver biopsy samples constructed by our center were assessed by the Affymetrix GeneChip using an Illumina Genome Analyzer at Novogene Corporation (Beijing, China). Briefly, mRNA was extracted from liver biopsies at the end of preservation and $1 \mathrm{~h}$ after revascularization. Reads with adapters, reads with undetermined base information and low-quality reads (reads with more than 50\% Qphred $\leq 20$ bases) were filtered. DEGs were identified using the Deseq R package (1.8.3), and p-values were adjusted using the Benjamini and Hochberg method. Transcripts with a fold change greater than 1.5 and an adjusted $p<0.05$ between two groups were considered significantly different. The gene ontology (GO) enrichment analysis of the DEGs was performed to explore the biological processes (BPs) involved, and the Goseq R package was applied to correct potential bias due to gene length. Pathway analysis was performed by enquiries into the Kyoto Encyclopedia of Genes and Genomes (KEGG) database (www.genome.jp/kegg/).

The full set of raw transcriptome data from this study has been deposited into the National Center for Biotechnology Information (NCBI) GEO database and is accessible via the GEO series accession number GSE113024.

\section{Gene-cloud of biotechnology information}

GCBI (Shanghai, China, www.gcbi.com.cn) is an online comprehensive bioinformatics analysis platform that combines a variety of research findings, genetic information, sample information, data algorithms and bioinformatics to create a 'gene knowledge base' that involves GEO datasets. The GCBI platform can systematically analyze GEO dataset-derived gene expression information, including more than 120 million copies of genomic samples [7]. In the present study, GCBI was used to identify DEGs between liver tissues postreperfusion and preimplantation. In the Differential Gene Expression Analysis module of the GCBI platform, we identified DEGs with a fold expression change $>1.5$ at cut-off values of $Q<0.05$ and $p<0.05$. Based on the DEGs, we further performed GO biological function analysis and KEGG pathway analysis. The top 20 biological functions and pathways are presented in tables. Venn diagrams were used to compare the top 100 DEGs from four chips, the top 20 KEGG pathways and the top 20 GO BPs using Venny (http://bioinfogp.cnb.csic.es/tools/venny/index.html). 


\section{Proteomic analysis}

Tissues were extracted from liver biopsies at the end of preservation and $1 \mathrm{~h}$ after revascularization. The samples were ground and digested by trypsin, and the peptides were desalted by a Strata X C18 SPE column (Phenomenex) and vacuum-dried. The peptides were then reconstituted in $0.5 \mathrm{M}$ TEAB and processed using the TMT kit according to the manufacturer's protocol. The tryptic peptides were fractionated by high $\mathrm{pH}$ reverse-phase HPLC. The peptides were subjected to nanospray ionization sourcing followed by MS/MS using Q Exactive ${ }^{T M}$ Plus (Thermo) coupled to the UPLC online. The peptides were then selected for MS/MS, and the resulting MS/MS data were processed using a Maxquant search engine (v.1.5.2.8). Based on relative quantification and statistical analysis, a 1.2-fold change and p-value $<0.5$ were set as the threshold for differentially abundance proteins (DAPs). Enrichment of GO and KEGG pathway analyses was consistent with the method described above. For each category of proteins, the InterPro database (a resource that provides a functional analysis of protein sequences by classifying them into families and predicting the presence of domains and important sites) was searched, and the two-tailed Fisher's exact test was employed to test the enrichment of the differentially expressed protein against all the identified proteins.

The full set of raw proteome data was deposited in ProteomeXchange via the PRIDE database (www.ebi.ac.uk/ pride) and is accessible through PXD010812.

\section{Transcriptome \& proteome cross-talk analysis}

In this study, we mainly studied the cross-talk relationship based on the quantification of the proteome and transcriptome, and proteins with a quantitative ratio above 1.2 or below $1 / 1.2$ and a t-test p-value $<0.05$ were deemed significantly differentially expressed. In transcriptome research, transcripts with a fold change above 1.5 and a corrected $\mathrm{p}$-value $<0.05$ were deemed significantly differentially expressed. Coregulated genes and proteins were identified and used for functional enrichment analysis. To obtain more biological information, we analyzed the protein functions of coregulated proteins or transcripts. GO, KEGG pathway and protein domain enrichment clustering analyses were performed, and the results are shown in the supplementary figure. Furthermore, the interactions of coregulated genes and coregulated proteins were analyzed by STRING (https://string-db.org/). GO, KEGG pathway and protein domain enrichment clustering analyses were performed based on the coregulated genes and coregulated proteins.

\section{Clinical tissue specimens, RNA extraction \& qRT-PCR analysis}

Samples from ten reperfused liver tissues and ten preimplanted liver tissues were utilized in our study to validate the expression levels of the coregulated DEGs. Prior patient consent and ethical approval from the ethics committee of the First Affiliated Hospital at Sun Yat-sen University were obtained. All methods were performed in accordance with the ethical guidelines and regulations. We selected ten coregulated DEGs for validation. Total RNAs from the tissue specimens were isolated using TRIzol reagent (Invitrogen, CA, USA), and qRT-PCR was performed using SYBR ${ }^{\circledR}$ Green dye (TaKaRa, Shiga, Japan) according to the manufacturer's instructions. The primer sequences are provided in Supplementary Table 1. $\beta$-tubulin was used as the reference gene.

\section{Ethics statement, ethical standards \& ethical approval}

The research protocol was reviewed and approved by the Research Ethics Committee of the First Affiliated Hospital at Sun Yat-sen University, and all experiments were conducted in accordance with guidelines approved by the First Affiliated Hospital at Sun Yat-sen University. All livers were procured at the Organ Transplant Center of The First Affiliated Hospital at Sun Yat-sen University. Informed consent was obtained from all individual participants included in the study. All procedures involving human participants were performed in accordance with the ethical standards of the First Affiliated Hospital at Sun Yat-sen University and the 1964 Helsinki Declaration and its later amendments or comparable guidelines. No tissues were procured from prisoners.

\section{Statistical analysis}

Student's t-test and analysis of variance were used to evaluate significant differences in demographic data. A Spearman $\chi^{2}$ test was used to identify correlations between gene and protein expression in the transcriptome and proteome. All p-values were two-sided, and $\mathrm{p}<0.05$ was defined as statistically significant. Analyses were carried out using Statistical Package for the Social Science (SPSS) 22.0 software (IBM, NY, USA). 


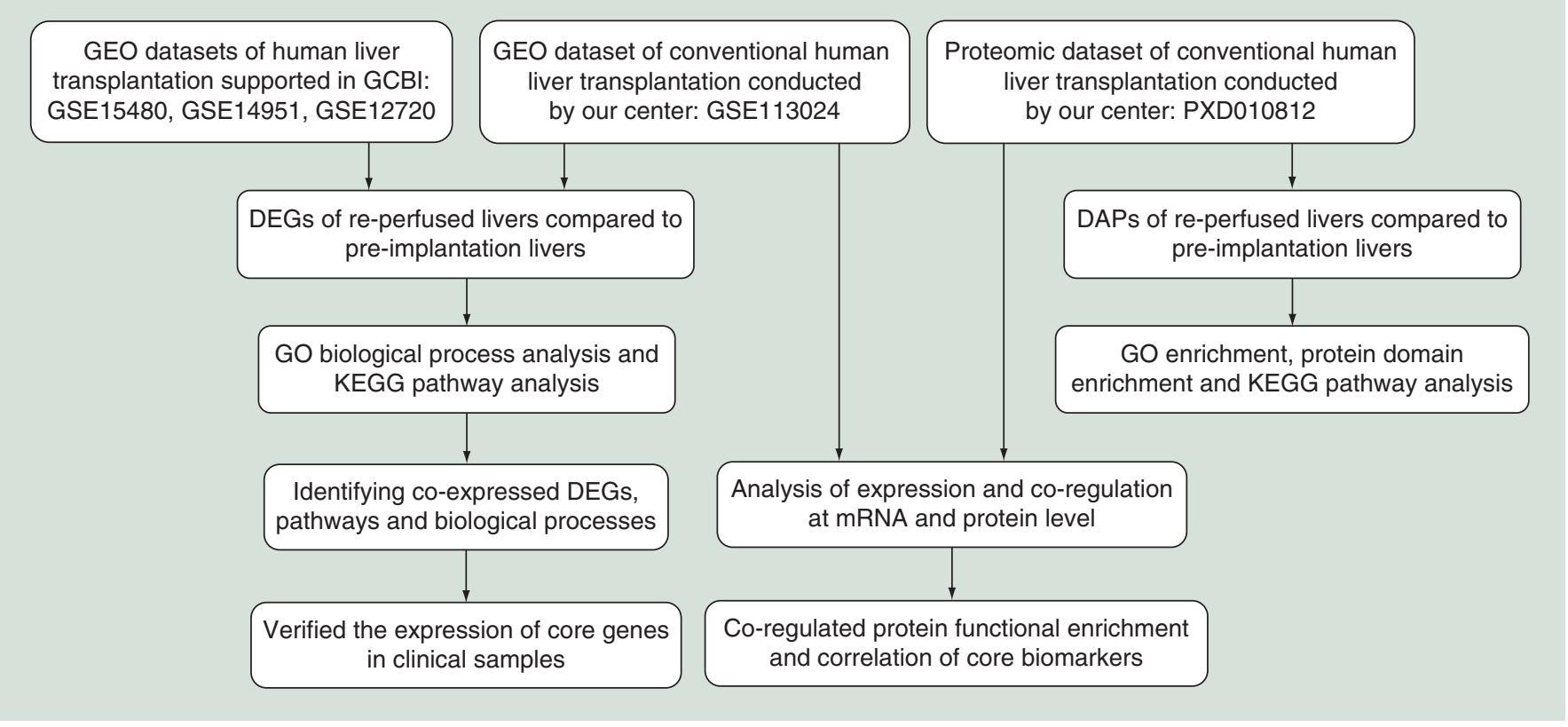

Figure 1. Flow diagram of the study design.

DAP: Differentially abundance protein; DEG: Differentially expressed gene; GCBI: Gene-cloud of biotechnology information; GEO: Gene Expression Omnibus; GO: Gene ontology; KEGG: Kyoto Encyclopedia of Genes and Genomes.

\section{Results}

Study design \& major characteristics of the dataset samples

Figure 1 shows the flow diagram of the study design. This study aimed to reveal key factors in liver allograft IRI, including core genes and proteins as well as biological functions identified by bioinformatics analysis. The GEO datasets GSE15480 (Chip 1), GSE14951 (Chip 2), GSE12720 (Chip 3) and GSE113024 (Chip 4) were enrolled in our study for transcriptome analysis. GSE15480 contained six preimplantation liver tissues and six postreperfusion liver tissues. GSE14951 contained five donor liver tissues and five transplanted-reperfused liver tissues. GSE12720 included 13 preimplantation deceased liver tissues and 13 reperfusion liver tissues. GSE113024, constructed by our center, contained three preimplantation liver tissues and three reperfusion liver tissues that were used for sequencing. The dataset PXD010812 (www.ebi.ac.uk/pride) subjected to proteomic and correlation analyses contained three preimplantation liver tissues and three reperfusion liver tissues, which were the same tissue sources as those of Chip 4. To further confirm the coregulated and coenriched results using transcriptomic datasets, correlation analysis of the transcriptome and proteome of CLT liver tissues was performed, and coregulated genes and proteins were identified. Coregulated gene and protein functional enrichment and correlation analyses of core biomarkers were conducted.

\section{DEGs between the reperfused \& preimplanted liver tissues}

We identified DEGs with a fold expression change $>1.5$ at cut-off values of $\mathrm{p}<0.05$. In total, 2183, 428, 341 and 1794 DEGs were identified in GSE15480, GSE14951, GSE12720 and GSE113024, respectively, as shown in the cluster heat map (Figure 2A-D) and volcano map (Figure 2F-I). After removing duplicate genes and those with expression values lacking specific gene symbols, the top ten DEGs from the four chips are shown in Supplementary Table 1. The top 100 DEGs from each chip were used to create a Venn diagram (Figure 3A). The intersection of DEGs in Figure 3A shows that ten genes were co-DEGs in all four chips, including ATF3, CCL4, DNAJB1, DUSP5, JUND, KLF6, NFKBIA, PLAUR, PPP1R15A and TNFAIP3. Five DEGs were coregulated in Chip 1, Chip 2 and Chip 3, including CCL3, ZC3H12A, FOSB, ICAM and JUN. Seven DEGs were coregulated in Chip 2, Chip 3 and Chip 4, including MXD1, GADD45B, BHLHE40, HSPA1A, SLC2A3, TRIB1 and BAG3. Two DEGs were coregulated in Chip 1, Chip 3 and Chip 4, including PLK3 and $M Y C$. Six DEGs were coregulated in Chip 1, Chip 2 and Chip 4, including SLC20A1, EMP1, WEE1, TIPARP, MAFF and CDKN1A. 
(A)

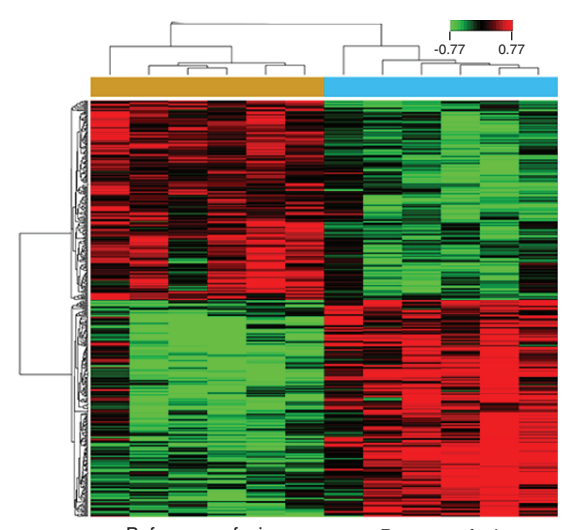

Before reperfusion Post reperfusion
(B)

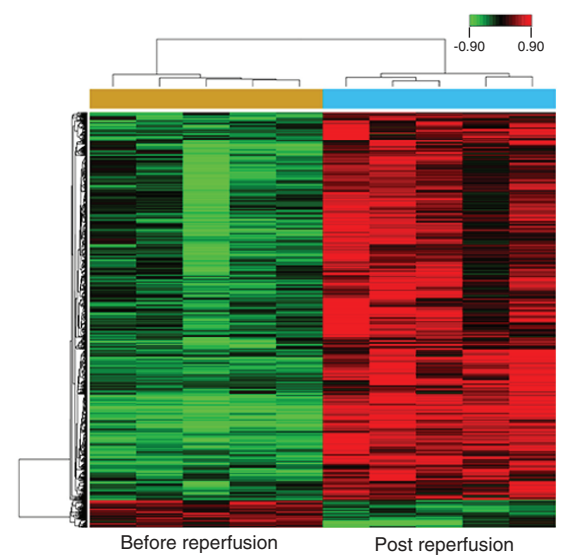

(c)

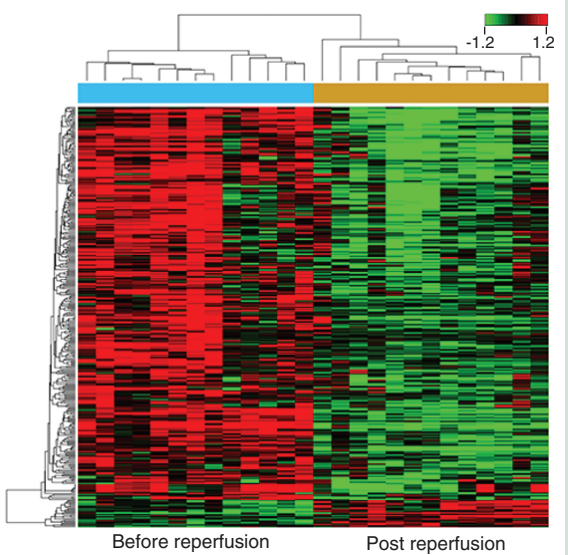

(D)

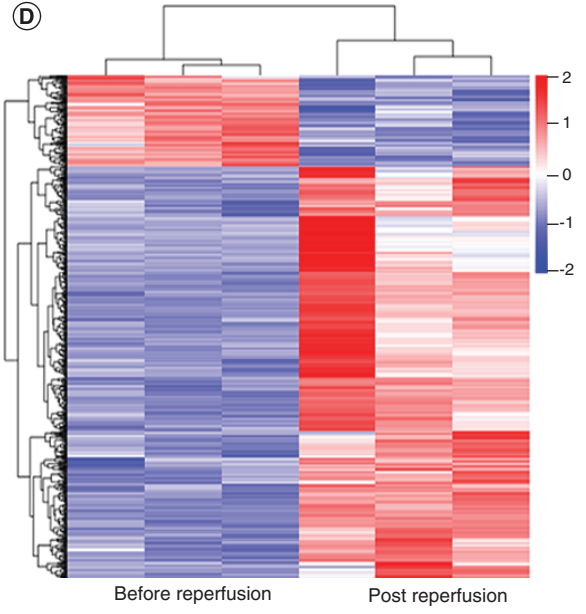

(E)

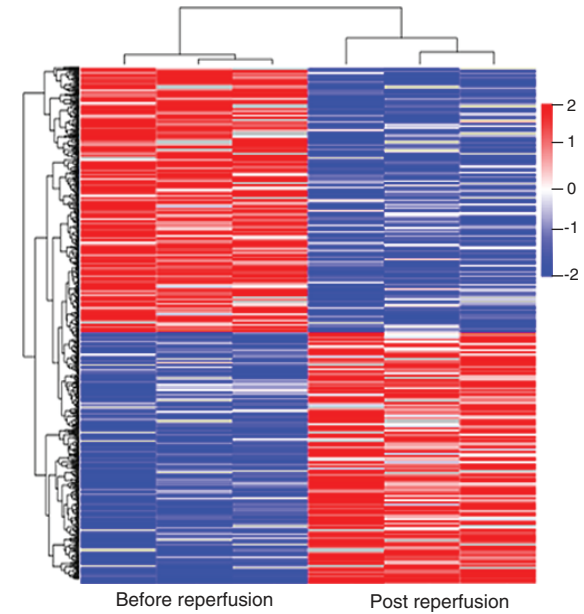

Figure 2. Differentially expressed genes and differentially abundance proteins. (A-D) Heat maps of potential differentially expressed genes between reperfusion and preimplantation liver tissues in four transcriptome datasets. (E) Heat map of potential differentially abundance proteins between reperfusion and preimplantation liver tissues in the proteome dataset. (F-I) Volcano plot of potential differentially expressed genes between reperfusion and preimplantation liver tissues in four transcriptome datasets. (J) Volcano plot of potential differentially abundance proteins between reperfusion and preimplantation liver tissues in the proteome dataset.

\section{Coenrichment analysis based on the transcriptome}

KEGG pathway analysis was used to investigate the pathways involving in which the identified DEGs were involed. The top 20 pathways from each chip, listed in Table 1, were used to create a Venn diagram (Figure 3B). The intersection of pathways in Figure 3B reveals that six pathways were coenriched in all four chips, including the 'NF-kappa B signaling pathway; NOD-like receptor signaling pathway; Influenza A; Legionellosis; Transcriptional misregulation in cancer; and MAPK signaling pathway'. Four pathways were coenriched in Chip 1, Chip 2 and Chip 3, including 'Pathways in cancer; Osteoclast differentiation; HTLV-I infection; and Chagas disease (American trypanosomiasis)'. Four pathways were coenriched in Chip 1, Chip 3 and Chip 4, including 'Malaria; Cytokine-cytokine receptor interaction; Rheumatoid arthritis; and African trypanosomiasis'. In the present study, GO analysis was used to identify the biological functions of the DEGs. BP analysis (Supplementary Table 2 \& Figure 3C) revealed that inflammatory response; negative regulation of cell proliferation; positive regulation of transcription, DNA-dependent; positive regulation of transcription from RNA polymerase II promoter; negative regulation of apoptotic process; signal transduction; and apoptotic process' were commonly enriched biological functions in Chip 1, Chip 2 and Chip 3. 'Angiogenesis' and 'response to lipopolysaccharide' were the common BPs in Chip1, Chip 2 and Chip 4. The three coenriched BPs in Chip 1 and Chip 3 were 'immune response; 

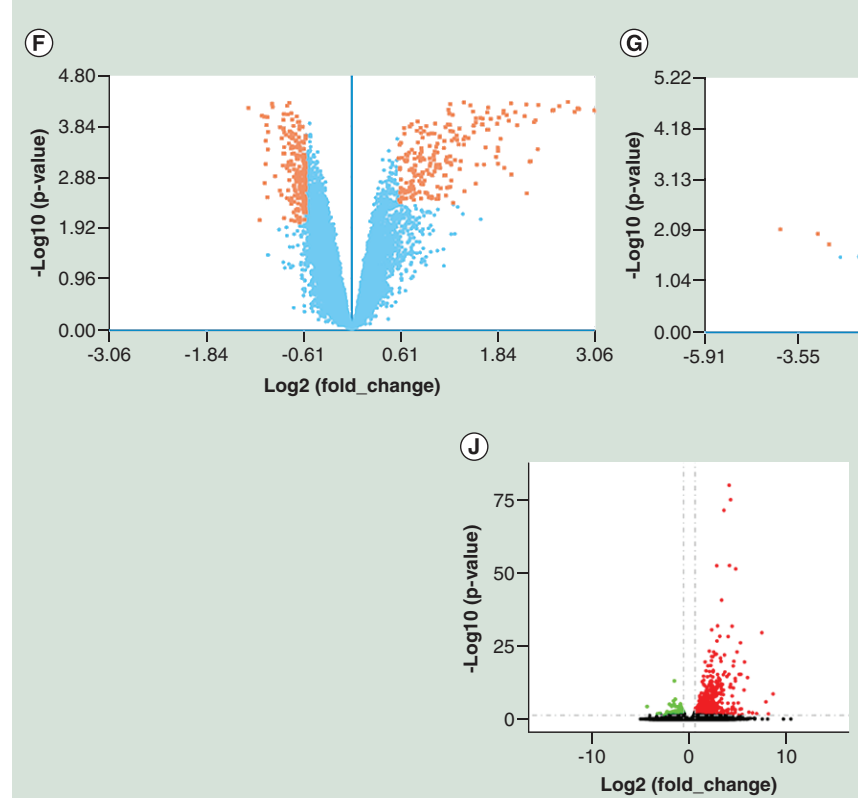

(a)

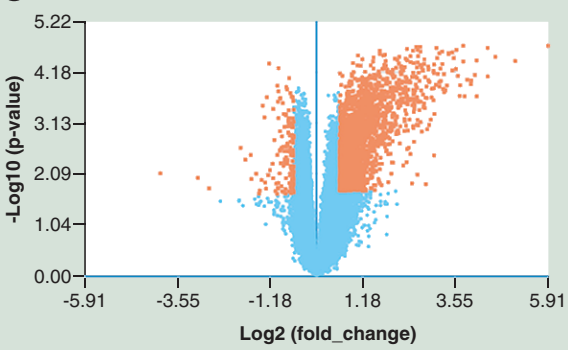

$(\mathbb{1}$

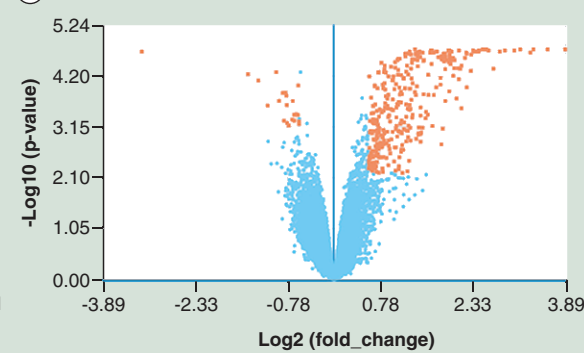

৫)

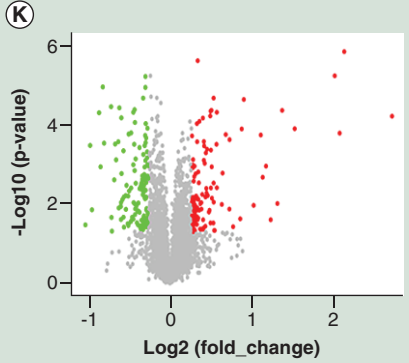

Figure 2. Differentially expressed genes and differentially abundance proteins (cont.). (A-D) Heat maps of potential differentially expressed genes between reperfusion and preimplantation liver tissues in four transcriptome datasets. (E) Heat map of potential differentially abundance proteins between reperfusion and preimplantation liver tissues in the proteome dataset. (F-I) Volcano plot of potential differentially expressed genes between reperfusion and preimplantation liver tissues in four transcriptome datasets. (J) Volcano plot of potential differentially abundance proteins between reperfusion and preimplantation liver tissues in the proteome dataset.

chemotaxis; and response to mechanical stimulus'. 'Transforming growth factor beta receptor signaling pathway' and 'transcription from RNA polymerase II promoter' were common BPs in Chip 2 and Chip 3, respectively.

\section{Validation of core gene expression in clinical samples}

We used real-time qPCR to detect the expression of ten coregulated DEGs, including ATF3, CCL4, DNAJB1, DUSP5, JUND, KLFG, NFKBIA, PLAUR, PPP1R15A and TNFAIP3, in clinical samples to further determine which genes might play a significant role in the progression of ischemia and reperfusion. Ten preimplantation liver tissue samples and ten reperfusion liver tissue samples were analyzed, and these ten coregulated DEGs were typically upregulated in the reperfused liver tissues compared with those in the preimplanted liver tissues $(\mathrm{p}<0.05)$, which was consistent with the results of the bioinformatics analysis described above (Figure 4A-J).

\section{Functional enrichment of DAPs between reperfused \& preimplanted liver tissues}

We identified DAPs with a fold change $>1.2$ at a cut-off value of $\mathrm{p}<0.05$. A total of 119 upregulated proteins and 125 downregulated proteins were identified, as shown in the cluster heat map (Figure 2E) and volcano map (Figure 2J). The top ten DAPs are shown in Table 1 and include $H B D, Z N F 648, F G A, H B B, F B N 1, D C N$, TF, IGHM, RPS15 and ROS1. Functional annotation of all the DAPs is shown in Figure 3. The GO terms (Figure 5A) are grouped into three categories (biological process, cellular component and molecular function). In the BP category, 'protein activation cascade' $-\log (\mathrm{p}$-value $)=24.66$ comprised the largest group, followed by 'defense response to bacterium' $-\log (\mathrm{p}$-value $)=12.95$ and 'humoral immune response' $-\log (\mathrm{p}$-value $)=12.86$. Protein domain enrichment (Figure 5B) revealed that 'leucine-rich repeat N-terminal domain; immunoglobulinlike fold; globin-like; TB domain; and globinprotoglobin and globin' were the most enriched. The DAPs are most likely involved in 'Complement and coagulation cascades; Retinol metabolism; Chemical carcinogenesis; Protein digestion and absorption; and Drug metabolism - cytochrome P450' as indicated by KEGG pathway enrichment analysis (Figure 5C). 


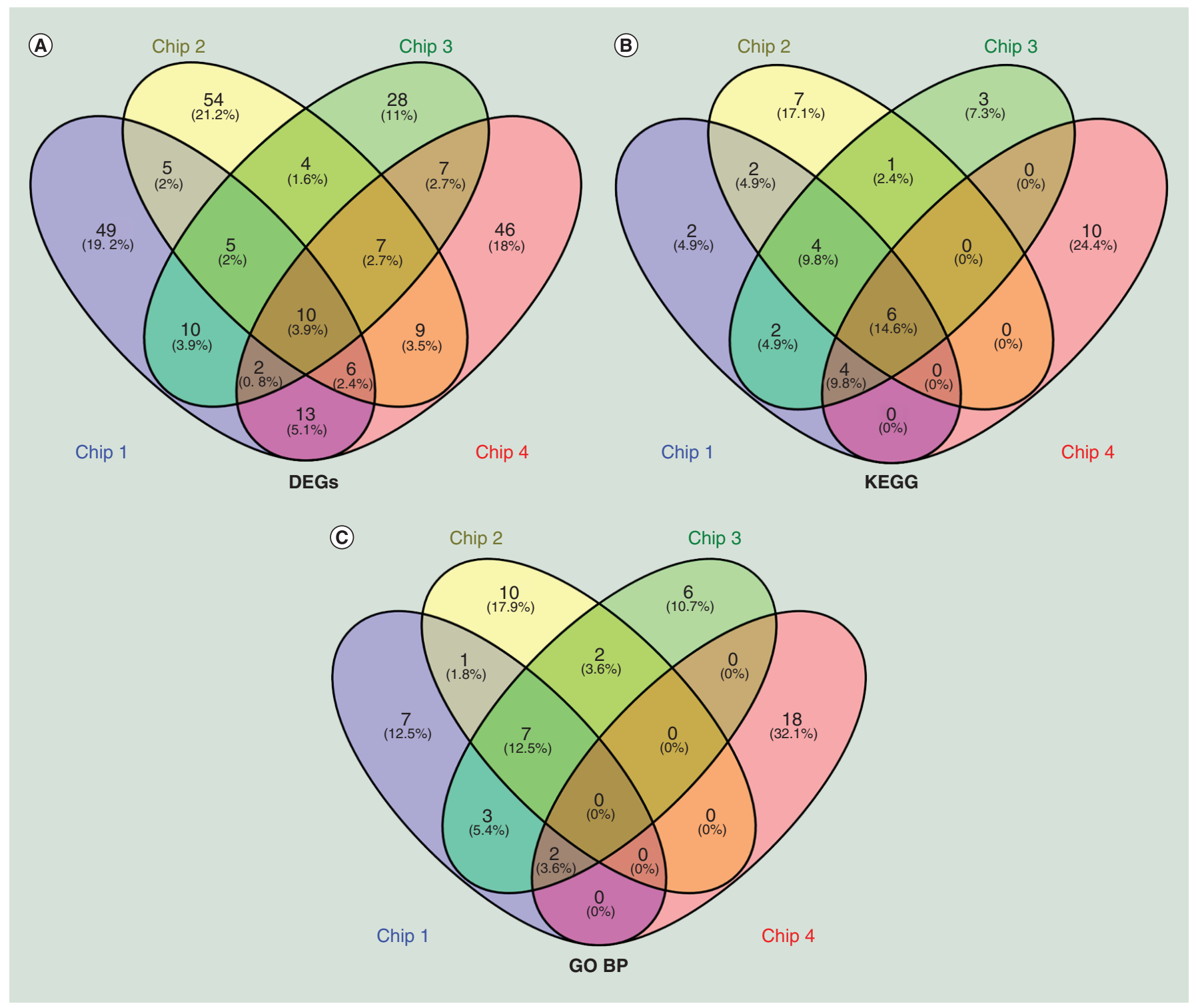

Figure 3. Coregulated differentially expressed genes and coenriched functions among four transcriptome datasets. (A) The Venn diagram shows the top 100 DEGs and coregulated genes. (B) The Venn diagram shows the top 20 KEGG pathways and coenriched pathways. (C) The Venn diagram shows the top 20 biological processes and coenriched processes.

BP: Biological processes; DEG: Differentially expressed gene; GO: Gene ontology; KEGG: Kyoto Encyclopedia of Genes and Genomes.

\section{Correlation analysis of the transcriptome \& proteome}

Next, we investigated the cross-talk relationship by quantifying the proteome and transcriptome. The results shown in Figure 6A revealed the correlation of the two omics. Upon comparing proteome and transcriptome datasets, 5536 proteins or transcripts were identified in both proteomic and transcriptomic data. Among these 5536 molecules identified, 17 genes and proteins were differentially expressed in both proteomic and transcriptomic data (Figure 6B). Among these biomarkers, ten were upregulated at both the mRNA and protein levels, including HBB, HBG2, CA1, SLC4A1, PLIN2, JUNB, HBA1, MMP9, SLC2A1 and PADI4 (Figure 6C). There were no codownregulated biomarkers. The functional enrichment cluster results of coregulated genes and proteins are shown in Supplementary Figure 1A-E, including GO analysis results (Supplementary Figure 1A-C), KEGG pathway analysis results (Supplementary Figure 1D) and protein domain analysis results (Supplementary Figure 1E). Then, the gene and protein interaction networks were analyzed by STRING (Figure 7). The network of coregulated DEGs and coregulation (proteome and transcriptome) revealed that ATF3, JUND, KLF6, NFKBIA, MMP9 and 


\begin{tabular}{|c|c|c|c|c|}
\hline Rank & Chip 1 & Chip 2 & Chip 3 & Chip 4 \\
\hline 1 & NF-kappa B signaling pathway & Spliceosome & NF-kappa B signaling pathway & IL-17 signaling pathway \\
\hline 2 & Malaria & MAPK signaling pathway & MAPK signaling pathway & TNF signaling pathway \\
\hline 3 & $\begin{array}{l}\text { Cytokine-cytokine receptor } \\
\text { interaction }\end{array}$ & Influenza A & Malaria & $\begin{array}{l}\text { Cytokine-cytokine receptor } \\
\text { interaction }\end{array}$ \\
\hline 4 & NOD-like receptor signaling pathway & Endocytosis & $\begin{array}{l}\text { Chagas disease (American } \\
\text { trypanosomiasis) }\end{array}$ & $\begin{array}{l}\text { Transcriptional misregulation in } \\
\text { cancer }\end{array}$ \\
\hline 5 & Rheumatoid arthritis & $\begin{array}{l}\text { Transcriptional misregulation in } \\
\text { cancer }\end{array}$ & Legionellosis & Malaria \\
\hline 6 & Influenza A & Legionellosis & $\begin{array}{l}\text { NOD-like receptor signaling } \\
\text { pathway }\end{array}$ & Legionellosis \\
\hline 7 & Pathways in cancer & NOD-like receptor signaling pathway & Rheumatoid arthritis & Fluid shear stress and atherosclerosis \\
\hline 8 & Legionellosis & HTLV-I infection & Osteoclast differentiation & NOD-like receptor signaling pathway \\
\hline 9 & Osteoclast differentiation & $\begin{array}{l}\text { Chagas disease (American } \\
\text { trypanosomiasis) }\end{array}$ & HTLV-I infection & FoxO signaling pathway \\
\hline 10 & Toll-like receptor signaling pathway & Epstein-Barr virus infection & Salmonella infection & $\begin{array}{l}\text { Complement and coagulation } \\
\text { cascades }\end{array}$ \\
\hline 11 & Salmonella infection & $\begin{array}{l}\text { Protein processing in endoplasmic } \\
\text { reticulum }\end{array}$ & Influenza A & $\begin{array}{l}\text { AGE-RAGE signaling pathway in } \\
\text { diabetic complications }\end{array}$ \\
\hline 12 & p53 signaling pathway & Osteoclast differentiation & $\begin{array}{l}\text { Cytokine-cytokine receptor } \\
\text { interaction }\end{array}$ & Influenza A \\
\hline 13 & $\begin{array}{l}\text { Transcriptional misregulation in } \\
\text { cancer }\end{array}$ & Hippo signaling pathway & $\begin{array}{l}\text { Transcriptional misregulation in } \\
\text { cancer }\end{array}$ & NF-kappa B signaling pathway \\
\hline 14 & MAPK signaling pathway & Hepatitis B & Chemokine signaling pathway & Rheumatoid arthritis \\
\hline 15 & HTLV-I infection & Herpes simplex infection & Hepatitis B & Bladder cancer \\
\hline 16 & Epstein-Barr virus infection & NF-kappa B signaling pathway & $\begin{array}{l}\text { Toll-like receptor signaling } \\
\text { pathway }\end{array}$ & MAPK signaling pathway \\
\hline 17 & $\begin{array}{l}\text { Protein processing in endoplasmic } \\
\text { reticulum }\end{array}$ & Insulin signaling pathway & Leishmaniasis & African trypanosomiasis \\
\hline 18 & $\begin{array}{l}\text { Chagas disease (American } \\
\text { trypanosomiasis) }\end{array}$ & Pathways in cancer & Toxoplasmosis & Insulin resistance \\
\hline 19 & African trypanosomiasis & PI3K-Akt signaling pathway & African trypanosomiasis & Prostate cancer \\
\hline 20 & Small cell lung cancer & Ubiquitin mediated proteolysis & Pathways in cancer & Estrogen signaling pathway \\
\hline
\end{tabular}

\begin{tabular}{|c|c|c|c|c|}
\hline \multirow[t]{2}{*}{ Name } & \multicolumn{2}{|c|}{ Transcriptome } & \multicolumn{2}{|c|}{ Proteome } \\
\hline & $\log 2$ (fold change) & p-value & $\log 2$ (fold change) & $\mathrm{p}$-value \\
\hline ATF3 & 4.484 & $7.64 \times 10^{-20}$ & - & - \\
\hline JUNB & 1.526 & $2.53 \times 10^{-7}$ & 1.448 & $8.87 \times 10^{-3}$ \\
\hline JUND & 2.785 & $3.81 \times 10^{-26}$ & 0.975 & $3.71 \times 10^{-1}$ \\
\hline KLF6 & 3.583 & $6.62 \times 10^{-24}$ & 1.056 & $2.31 \times 10^{-1}$ \\
\hline MMP9 & 1.924 & $1.24 \times 10^{-7}$ & 1.305 & $1.33 \times 10^{-2}$ \\
\hline NFKBIA & 1.851 & $2.80 \times 10^{-12}$ & 1.085 & $5.53 \times 10^{-1}$ \\
\hline
\end{tabular}

$J U N B$ are the core biomarkers building up the core gene connections. The regulation of the core genes and proteins is shown in Table 2.

According to the functional enrichment results (Table 3), the core genes/proteins are closely related to the 'regulation of cell death; negative regulation of signal transduction; and response to inorganic substance', as these terms were enriched in the BP GO analysis category. The KEGG pathway results showed that these processes are involved in the 'TNF signaling pathway and the NF-kappa B signaling pathway'. Protein domains were enriched for 'Globin', and molecular functions were enriched for 'oxygen transporter activity'. 
(A)

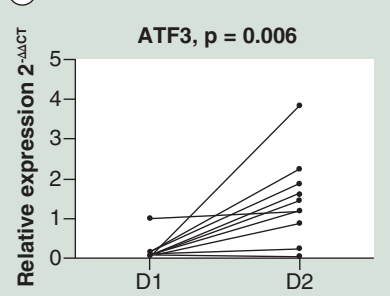

(E)

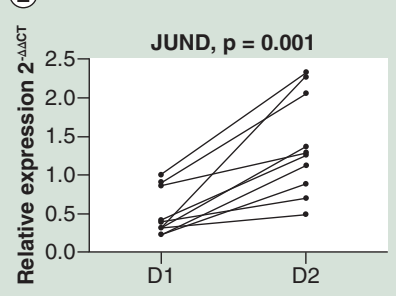

(B)

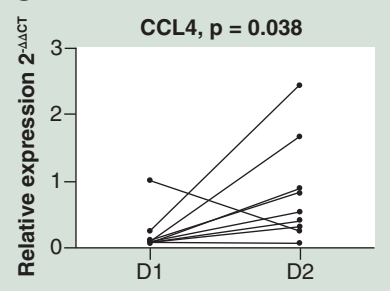

(F)

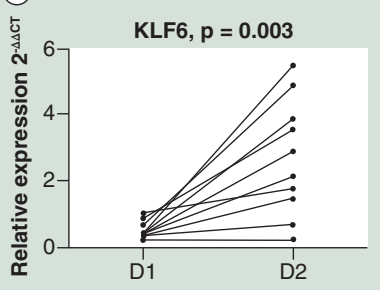

(1)

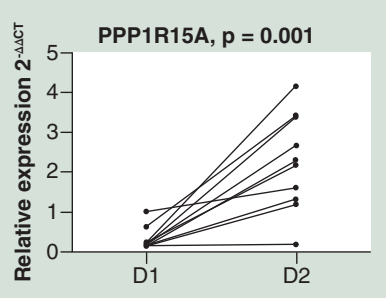

(C)

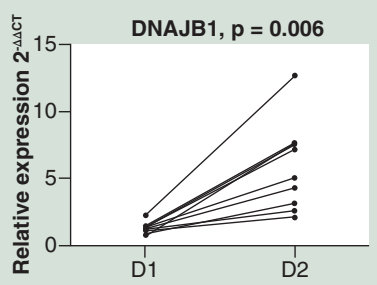

(a)

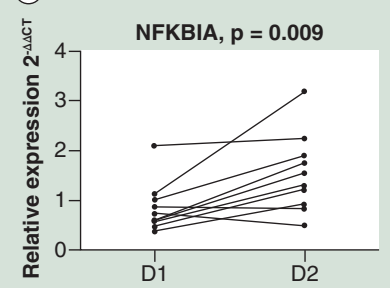

(D)

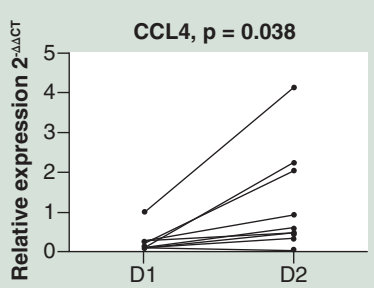

(H)

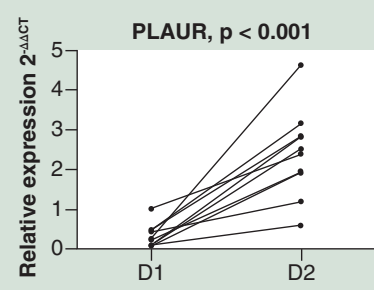

(I)

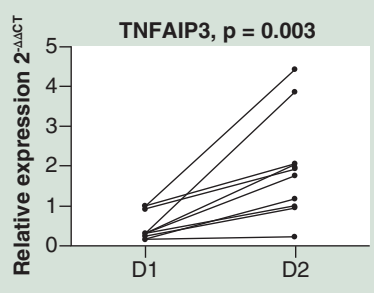

Figure 4. Validation of differentially expressed genes. (A-J) Real-time quantitative PCR validation of ten coregulated differentially expressed genes in ten paired preimplantation and reperfusion liver tissues.

\section{Discussion}

Liver IRI is a clinically relevant phenomenon that occurs in a wide range of settings, including trauma surgery, hepatic resection and transplantation, and affects clinical outcomes [8]. Liver IRI has been extensively studied in recent decades and implicated in the pathophysiologies of many clinical entities [9]. A complex interplay exists between cellular mediators, the complement system, cytokines/chemokines and other secreted factors that activates several parallel intracellular pathways that include transcription factors and nitric oxide synthase [8]. IRI can also activate the immune system, thus driving the full development of inflammatory hepatocellular injury [10]. Molecular processes occurring during hepatic IRI are diverse and continuously include new and complex mechanisms, all of which are beginning to be unravelled.

With the rapid development of omics, studies on the pathophysiologies of various clinical issues have been pushed to higher levels and include combining multiple forms of omics data into an integrated framework [11]. A better understanding of the mechanisms underlying IRI will lead to novel therapies for relieving liver IRI-related morbidity and mortality $[1,12]$. In this study, we imported three GEO datasets as well as a GEO dataset constructed by our center into the GCBI comprehensive analysis platform to extract liver IRI gene expression data.

This study is the first to perform a comprehensive analysis of the transcriptome and proteome of human liver IRI. As shown in Figure 2, in the four gene arrays, substantial numbers of genes were significantly up- or downregulated in liver tissues after reperfusion, indicating a fierce attack on the liver caused by IRI. We identified coregulated DEGs between preimplantation and postreperfusion liver samples and found that ATF3, CCL4, DNAJB1, DUSP5, JUND, KLFG, NFKBIA, PLAUR, PPP1R15A and TNFAIP3 were coregulated DEGs in all four Chips and could thus be key genes in the pathophysiological process of IRI. These results were solidly validated in a large number of clinical samples (Figure 4). Moreover, we conducted a proteomic analysis of liver IRI to identify DAPs and functional enrichment. It would take a longer process for proteomic to present a significant change, resulting in a low correlation of transcriptomic and proteomic analysis. The combined omics analysis identified 


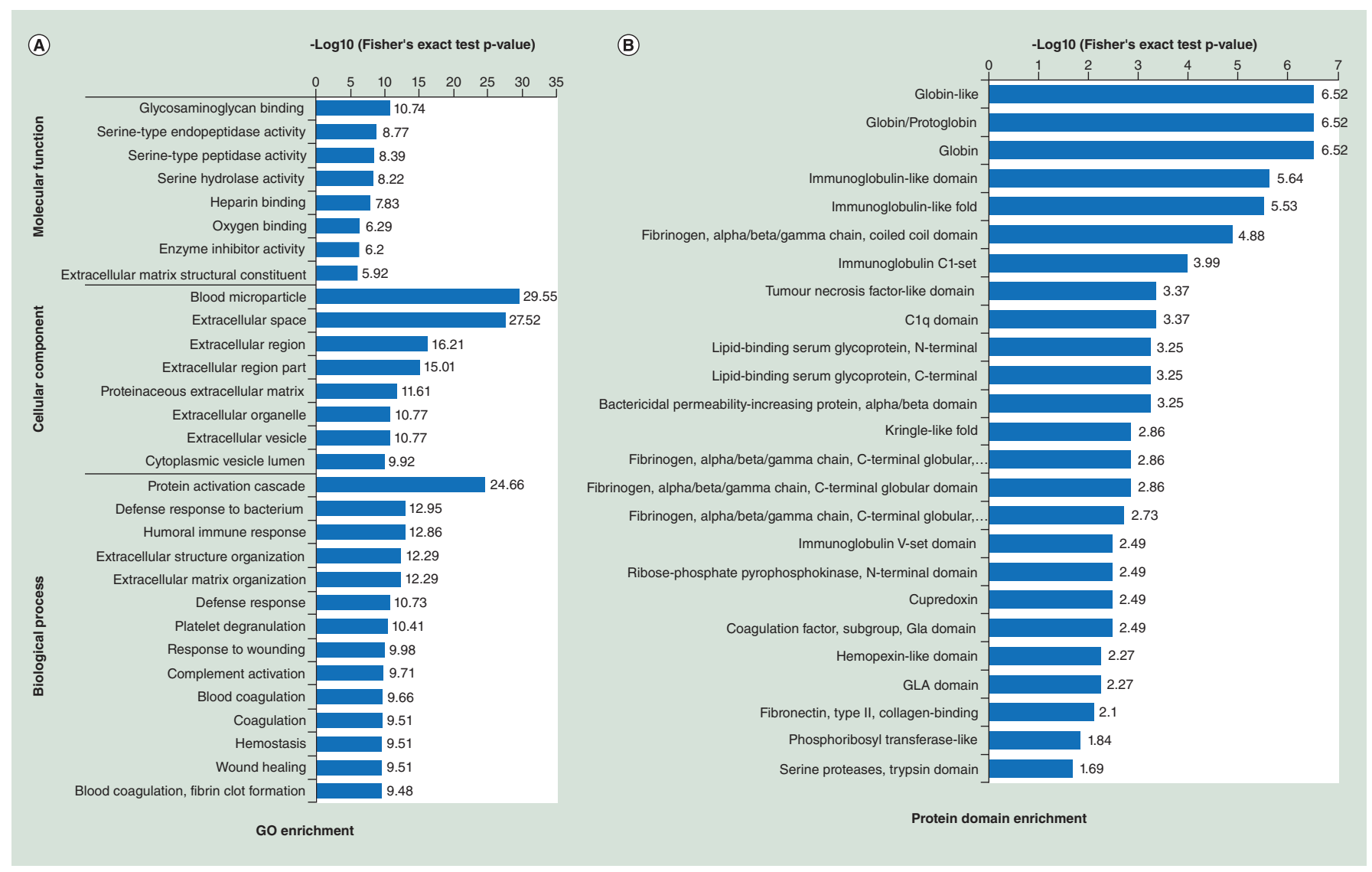

Figure 5. Functional enrichment of liver ischemia-reperfusion injury as indicated by the proteome. (A) GO enrichment analysis. (B) Protein domain enrichment analysis. (C) KEGG pathway enrichment analysis.

GO: Gene ontology.

some genes and proteins that might play a vital role in liver IRI and elucidated ten additional proteins that were co-upregulated. Together, the coregulated genes and coregulated proteins comprise the core factors underlying liver IRI (Figure 7). Some of the coregulated DEGs have been reported to be closely related to liver IRI. Rao et al. [13] found that ATF3 significantly increased ischemia/reperfusion (IR)-stressed liver injury, as evidenced by increased serum alanine aminotransferase levels, histological liver damage and hepatocellular apoptosis. ATF3mediated NRF2/HO-1 signaling in the regulation of TLR4-driven inflammatory responses in IR-stressed livers. The protein level of TNFAIP3 (A20) is reportedly increased after reperfusion, which could aggravate postischemic liver injury [14]. Other coregulated DEGs have also been studied in various IR models. CCL4, DNAJB1 and $J U N D$ are highly upregulated in postischemic myocardia and mouse hippocampal tissues after transient forebrain ischemia as identified by microarray analysis, and the long noncoding RNA H19 induces cerebral IRI via the DUSP5-ERK1/2 axis to active autophagy [15]. Lin et al. [16] showed that KLF6 is obviously upregulated and may play a role in the pathogenesis of acute kidney injury. PLAUR regulation by $M y b$ and Fos might participate in cortex and striatum injury after cerebral IR [17], and PLAUR is of substantial importance in human microsurgical-free muscle tissue transfer [18]. Moreover, endoplasmic reticulum stress-induced Paneth cell apoptosis contributes to intestinal IR-induced bacterial translocation and systemic inflammation, and the induction of DNA damage by PPP1R15A could play a vital role in this process [19]. There was no doubt that regulated (but not coregulated) genes and proteins were also related to IRI process to some extent. Some genes, including coexpressed genes, have been proven to be potential targets to reduce IRI in animal experiments, including the coexpressed genes [20]. However, there was no evidence of a correlation for these genes with clinical outcome. Further analysis on clinical outcomes is of great significance.

The intersection of KEGG pathways revealed that six pathways were coenriched in all four chips, including the 'NF-kappa B signaling pathway; NOD-like receptor signaling pathway; influenza A; legionellosis; transcriptional 


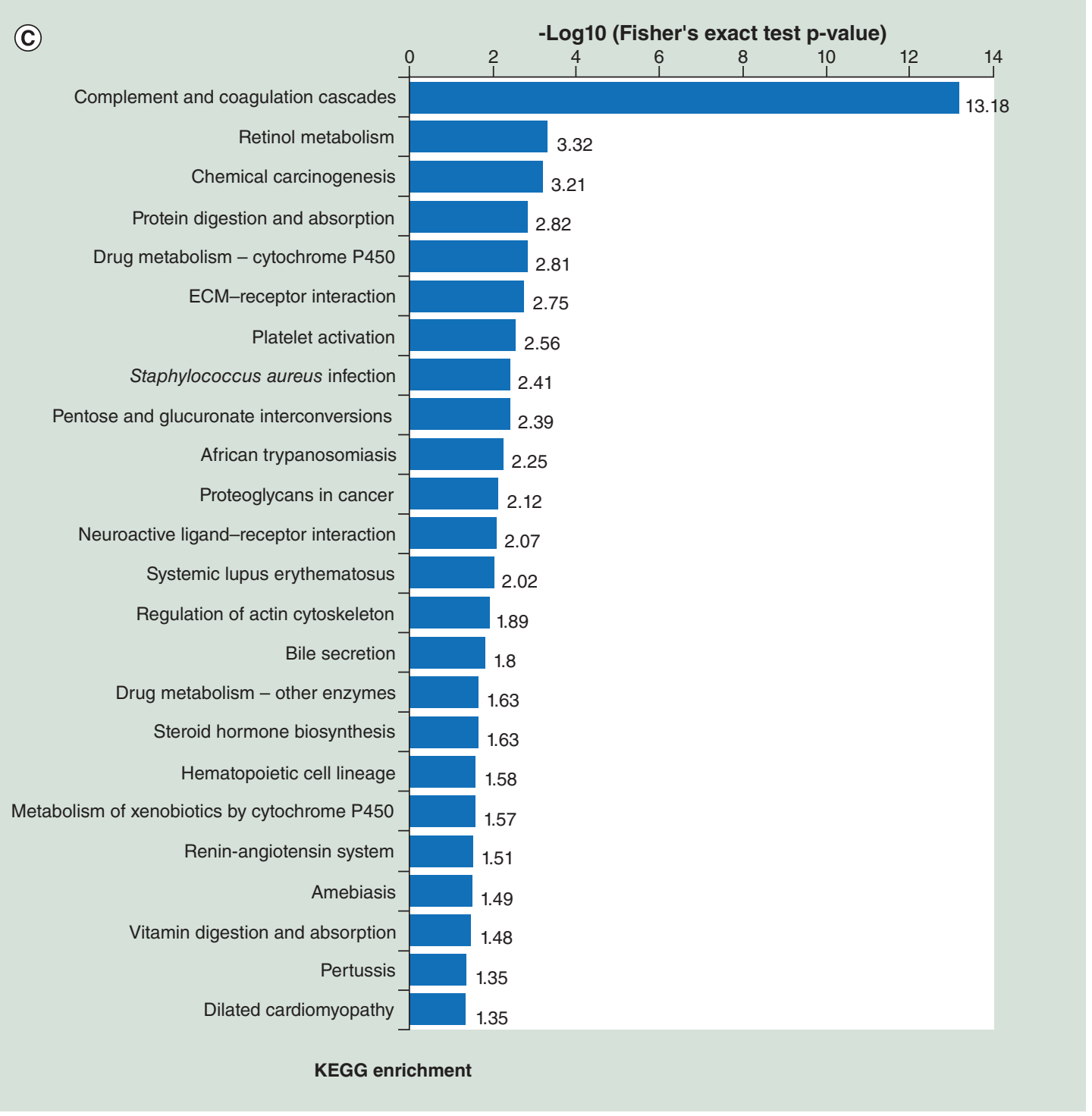

Figure 5. Functional enrichment of liver ischemia-reperfusion injury as indicated by the proteome (cont.). (A) GO enrichment analysis. (B) Protein domain enrichment analysis. (C) KEGG pathway enrichment analysis. GO: Gene ontology.

misregulation in cancer; and MAPK signaling pathway'. These pathways have been reported to be vital in liver IRI [21-24]. NF- $\mathrm{B}$ activation in hepatocytes and macrophages appeared as a double-edged-sword in hepatic IRI [25]. Treatments targeting NF- $\mathrm{KB}$ may be a viable therapeutic method in acute liver injury [23]. NOD-like receptors' signaling pathway plays important role in cellular proliferation. Liver IRI could activate NOD-like receptors and promote liver regeneration in clinical [26]. 'Influenza A; legionellosis' could unlikely to be correlated to IRI. MAPK signaling pathway contributes to inflammation that can be either beneficial or detrimental to the liver [27].

Proteomic analysis was previously performed using human orthotopic liver transplantation samples to identify early molecular events and cell metabolism reprogramming that occurs upon IR. All of the studies also tried to identify key proteins. Emadali et al. performed a global patterns of expression and phosphorylation of cytoskeletonrelated proteins and a functional profiling of the tyrosine phosphoproteome during liver transplantation [28,29]. Vascotto et al. conducted a proteomic analysis of only 800-900 protein spots by using 2-DE and MALDI-TOF MS [30]. Our very study had a global analysis of abundance of more than 5000 proteins. This is also the first report of using a combined transcriptomic and proteomic analysis of human liver IRI samples, and the deeper landscape of the mechanism underlying liver IRI has been revealed to some extent. Correlation analysis of the transcriptome 
(A)

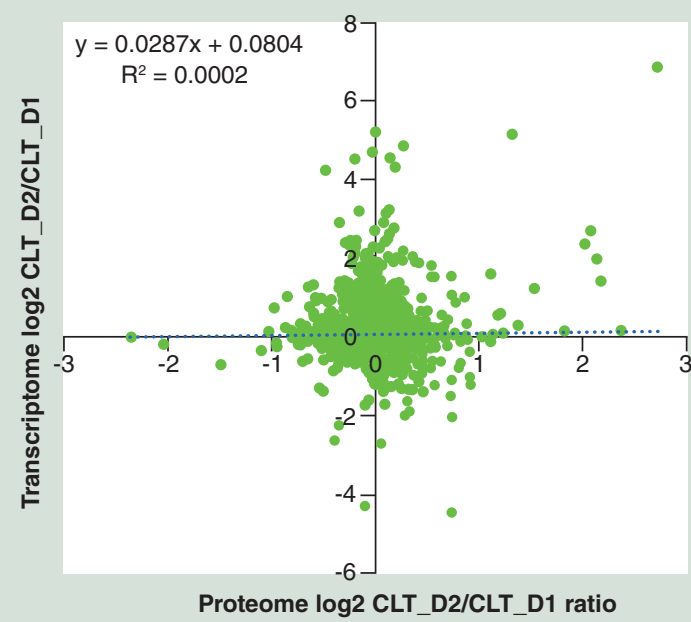

(B)

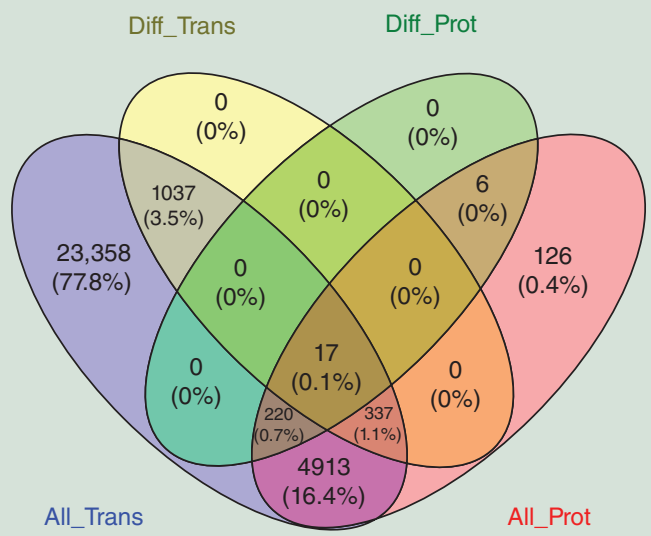

(C)

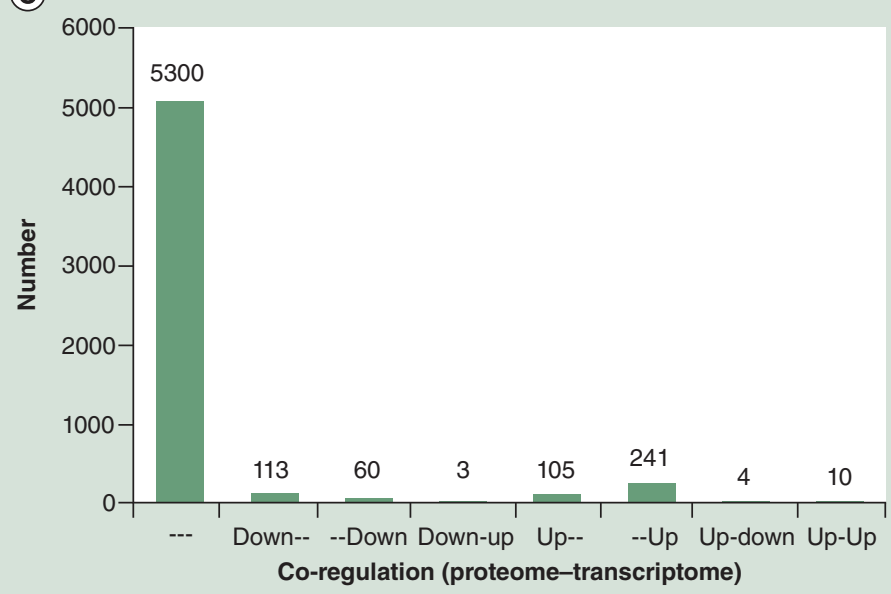

Figure 6. Transcriptome and proteome correlation analysis. (A) Quantification of the transcriptome and proteome relationship. (B) Cross-talk was identified in both proteomic and transcriptomic data. (C) Differential regulation of genes and proteins in the proteome and transcriptome.

and proteome demonstrated ten co-upregulated proteins that were closely related to the ten coregulated DEGs, as indicated by the interaction network elucidated. Their enriched functions, such as the 'TNF signaling pathway and NF-kappa B signaling pathway', were reported to be key factors in IRI [1,31]. Among these pathways, ATF3, $J U N D, K L F 6, N F K B I A, M M P 9$ and JUNB are the core factors and participate in key functions. For instance, all these six genes in the network closely interacted with the NF-kappa B signaling pathway, all of which could be a gene bloc processing liver IRI $(12076863,28166199,29650964,17255940)$. The core genes could also participate in various signaling pathways. Inhibitory or promoting treatments targeting these genes would greatly affect the progress of liver IRI, which suggests that such treatments are promising methods for studies aiming to relieve liver IRI.

\section{Conclusion \& future perspective}

In the present study, a tandem transcriptomic and proteomic profiling approach was utilized to elucidate the differences between preimplanted and reperfused livers. Our research provides a comprehensive overview of the proteins/genes involved in the process of IRI during liver transplantation. This study will be helpful for identifying the core genes and proteins involved in IRI and understanding the mechanism underlying this process. Moreover, 


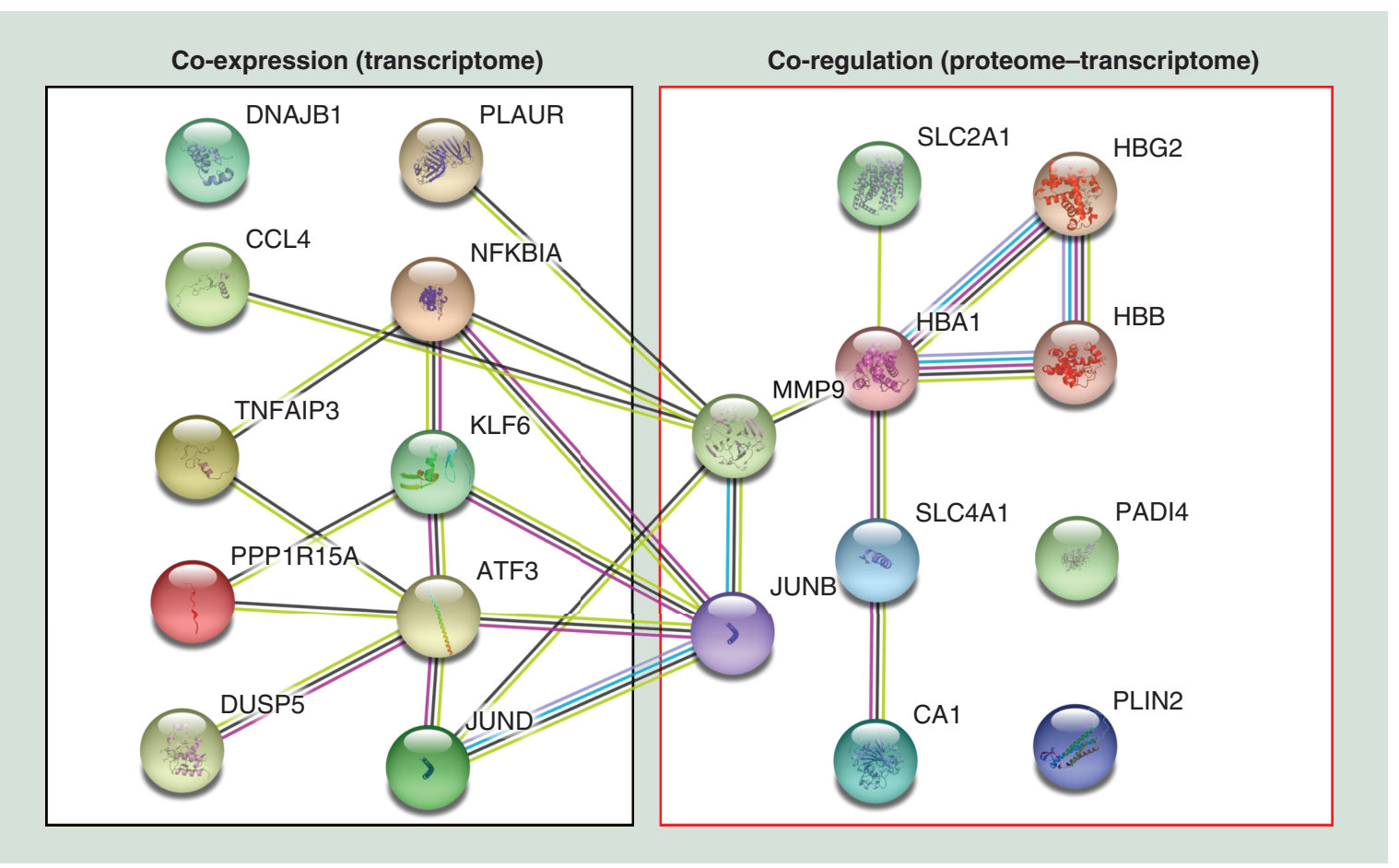

Figure 7. The interaction network of the coregulated genes and coregulated proteins.

\begin{tabular}{|c|c|c|}
\hline Enrichments & Gene count & Matching genes/proteins \\
\hline \multicolumn{3}{|l|}{ Biological process } \\
\hline Bicarbonate transport & 4 & CA1, HBA1, HBB, SLC4A1 \\
\hline Response to inorganic substance & 7 & CD9, HBA1, HBB, JUNB, JUND, KLF6, TNFAIP3 \\
\hline Oxygen transport & 3 & HBA1, HBB, HBG2 \\
\hline Organic anion transport & 6 & APOA1, CA1, HBA1, HBB, PLIN2, SLC4A1 \\
\hline Regulation of cell death & 10 & ATF3, HBA1, HBB, JUNB, JUND, MMP9, NAT8, NFKBIA, PLAUR, TNFAIP3 \\
\hline Response to hydrogen peroxide & 4 & HBA1, HBB, KLF6, TNFAIP3 \\
\hline $\begin{array}{l}\text { Negative regulation of cysteine-type endopeptidase activity involved } \\
\text { in apoptotic signaling pathway }\end{array}$ & 2 & MMP9, PLAUR \\
\hline Negative regulation of signal transduction & 8 & APOA1, ATF3, DUSP5, MMP9, NFKBIA, PLAUR, PPP1R15A, TNFAIP3 \\
\hline \multicolumn{3}{|l|}{ KEGG pathway } \\
\hline TNF signaling pathway & 4 & JUNB, MMP9, NFKBIA, TNFAIP3 \\
\hline African trypanosomiasis & 3 & APOA1, HBA1, HBB \\
\hline NF-kappa B signaling pathway & 3 & CCL4, NFKBIA, TNFAIP3 \\
\hline Osteoclast differentiation & 3 & JUNB, JUND, NFKBIA \\
\hline \multicolumn{3}{|l|}{ Protein domains } \\
\hline Globin & 3 & HBA1, HBB, HBG2 \\
\hline \multicolumn{3}{|l|}{ Molecular function } \\
\hline Oxygen transporter activity & 3 & HBA1, HBB, HBG2 \\
\hline \multicolumn{3}{|l|}{ Cellular component } \\
\hline Endocytic vesicle lumen & 3 & APOA1, HBA1, HBB \\
\hline Haptoglobin-hemoglobin complex & 2 & HBA1, HBB \\
\hline Blood microparticle & 4 & APOA1, HBA1, HBG2, SLC4A1 \\
\hline Endocytic vesicle & 4 & APOA1, CD9, HBA1, HBB \\
\hline
\end{tabular}


this study constitutes the first comprehensive analysis of the genetic information presented during IRI and the first correlation analysis of the IRI transcriptome and proteome. Our study will substantially advance the molecular research on and functional identification of IRI, thereby providing a deeper understanding of the mechanisms underlying this process. Clinicians would also greatly benefit from knowing more details on the preservation methods and immunosuppressive regimen according to this study. This knowledge may be of great significance for reducing the adverse impacts of IRI in clinical practice.

\section{Summary points}

- This study identified that ten differentially expressed genes (DEGs) were co-upregulated in the four Gene Expression Omnibus datasets referring to ischemia-reperfusion injury (IRI) in liver transplantation, including ATF3, CCL4, DNAJB1, DUSP5, JUND, KLF6, NFKBIA, PLAUR, PPP1R15A and TNFAIP3.

- Coexpression of the DEGs in ten matched clinical samples was verified by quantitative real-time PCR. According to previous study, the coexpressed genes could play vital roles in liver IRI and be therapeutic targets to reduce injury from ischemia and reperfusion.

- Six pathways were coenriched in all four chips, including the NF-kappa B signaling pathway; NOD-like receptor signaling pathway; Influenza A; Legionellosis; Transcriptional misregulation in cancer; and MAPK signaling pathway. Most of them were proved to be important pathways involved in IRI.

- The combined analysis demonstrated ten coregulated genes/proteins, including HBB, HBG2, CA1, SLC4A1, PLIN2, JUNB, HBA1, MMP9, SLC2A1 and PADI4.

- The coregulated DEGs and coregulated genes/proteins formed a tight interaction network and could serve as the core factors underlying IRI. Further study on the genes and proteins could reveal the core process underlying IRI during human liver transplantation.

Supplementary data

To view the supplementary data that accompany this paper please visit the journal website at: https://www.futuremedicine.com/d oi/suppl/10.2217/epi-2018-0189

\section{Author's contributions}

SZ Huang conceived and designed the study; M Han, HT Chen and FX Lin helped with the revision; RX Xie, ZB Zhu, WQ Ju and DP Wang searched databases; YH Tang, ZH Zhang, J Yang, Q Zhao and MG Chen performed data analyses; CJ Sun, YX Zhang, RX Xie, CH Zhu prepared the tables and figures; SZ Huang, Q Zhou, ZY Guo and XS He wrote and revised the manuscript; FX Lin and HT Chen helped with revision. All the authors reviewed the manuscript.

Financial \& competing interests disclosure

This study was supported by the following grants: the National Natural Science Foundation of China (81373156 and 81570587), the Guangdong Provincial International Cooperation Base of Science and Technology (Organ Transplantation; 2015B050501002), the Science and Technology Program of Guangzhou (201704020150) and the Science and Technology Program of Huizhou (170520181743174/2017Y229 and 180529101741637/2018Y305). The authors have no other relevant affiliations or financial involvement with any organization or entity with a financial interest in or financial conflict with the subject matter or materials discussed in the manuscript apart from those disclosed.

No writing assistance was utilized in the production of this manuscript.

\section{References}

Papers of special note have been highlighted as: $\bullet$ of interest; $\bullet \bullet$ of considerable interest

1. Eltzschig HK, Eckle T. Ischemia and reperfusion - from mechanism to translation. Nat. Med. 17, 1391-1401 (2011).

-. Thoroughly reviewed the mechanism and translation practice of ischemia and reperfusion.

2. Vollmar B, Menger MD. The hepatic microcirculation: mechanistic contributions and therapeutic targets in liver injury and repair. Physiol. Rev. 89, 1269-1339 (2009).

3. Nemes B, Gaman G, Polak WG et al. Extended-criteria donors in liver transplantation part II: reviewing the impact of extended-criteria donors on the complications and outcomes of liver transplantation. Expert. Rev. Gastroenterol. Hepatol. 10, 841-859 (2006).

4. Liu H, Jayakumar S, Traboulsi M et al. Cirrhotic cardiomyopathy: implications for liver transplantation. Liver Transpl. 23, 826-835 (2017). 
5. He X, Guo Z, Zhao Q et al. The first case of ischemia-free organ transplantation in humans: a proof of concept. Am. J. Transplant. 18, 737-744 (2018).

-• First clinical method to completely avoid ischemia-reperfusion injury in liver transplantation.

6. Kahles A, Lehmann KV, Toussaint NC et al. Comprehensive analysis of alternative splicing across tumors from 8,705 patients. Cancer Cell 34, 211-224.e6 (2018).

- Describing a comprehensive analysis of omics with a huge group of samples.

7. Huang S, Sun C, Hou Y et al. A comprehensive bioinformatics analysis on multiple gene expression omnibus datasets of nonalcoholic fatty liver disease and nonalcoholic steatohepatitis. Sci. Rep. 8, 7630 (2018).

8. Datta G, Fuller BJ, Davidson BR. Molecular mechanisms of liver ischemia reperfusion injury: insights from transgenic knockout models. World J. Gastroenterol. 19, 1683-1698 (2013).

9. Nastos C, Kalimeris K, Papoutsidakis N et al. Global consequences of liver ischemia/reperfusion injury. Oxid. Med. Cell. Longev. 2014, 906-965 (2014).

10. Lu L, Zhou H, Ni M et al. Innate immune regulations and liver ischemia-reperfusion injury. Transplantation 100, 2601-2610 (2016).

11. Franzosa EA, Hsu T, Sirota-Madi A et al. Sequencing and beyond: integrating molecular 'omics' for microbial community profiling. Nat. Rev. Microbiol. 13, 360-372 (2015).

12. Zhang C, Huang J, An W. Hepatic stimulator substance resists hepatic ischemia/reperfusion injury by regulating Drp1 translocation and activation. Hepatology 66, 1989-2001 (2017).

13. Rao J, Qian X, Li G et al. ATF3-mediated NRF2/HO-1 signaling regulates TLR4 innate immune responses in mouse liver ischemia/reperfusion injury. Am. J. Transplant. 15(1), 76-87 (2015).

14. Yu J, Lee HS, Lee SM et al. Aggravation of post-ischemic liver injury by overexpression of A20, an NF-kappaB suppressor. J. Hepatol. 55, 328-336 (2011).

15. Wang J, Cao B, Han D et al. Long non-coding RNA H19 induces cerebral ischemia reperfusion injury via activation of autophagy. Aging Dis. 8(1), 71-84 (2017).

16. Lin J, Mao X, Wu H et al. Genes expression in the early stage of acute renal ischemia-reperfusion injury in rats. Fa Yi Xue Za Zhi 32 , 401-405 (2016).

17. Zhao Z, Lu Z, Sun X et al. Global transcriptomic profiling of cortex and striatum: cerebral injury after ischemia/reperfusion in a mouse model. J. Stroke Cerebrovasc. Dis. 26, 1622-1634 (2017).

18. Dragu A, Schnurer S, Surmann-Schmitt C et al. Gene expression analysis of ischaemia and reperfusion in human microsurgical free muscle tissue transfer. J. Cell. Mol. Med. 15, 983-993 (2011).

19. Grootjans J, Hodin CM, de Haan JJ et al. Level of activation of the unfolded protein response correlates with Paneth cell apoptosis in human small intestine exposed to ischemia/reperfusion. Gastroenterology 140, 529-539.e3 (2011).

20. Selzner N, Rudiger H, Graf R, Clavien PA. Protective strategies against ischemic injury of the liver. Gastroenterology 125(3), 917-936 (2003).

- An important review describing methods that have been done to relieve ischemic injury of the liver.

21. Murrow L, Debnath J. Autophagy as a stress-response and quality-control mechanism: implications for cell injury and human disease. Annu. Rev. Pathol. 24(8), 105-137 (2013).

22. Ocuin LM, Zeng S, Cavnar MJ et al. Nilotinib protects the murine liver from ischemia/reperfusion injury. J. Hepatol. 57, 766-773 (2012).

23. Sakai N, Van Sweringen HL, Schuster R et al. Receptor activator of nuclear factor-kappaB ligand (RANKL) protects against hepatic ischemia/reperfusion injury in mice. Hepatology 55, 888-897 (2012).

- A very interesting study finding that RANKL can protect liver ischemia/reperfusion injury by NF- $\mathrm{KB}$ in mice.

24. Zheng X, Zhou H, Qiu Z et al. Gene microarray analysis of expression profiles in liver ischemia and reperfusion. Mol. Med. Rep. 16, 3299-3307 (2017).

25. Hou J, Xia Y, Jiang R et al. PTPRO plays a dual role in hepatic ischemia reperfusion injury through feedback activation of NF- $\kappa \mathrm{B}$. J. Hepatol. 60(2), 306-312 (2014).

26. Lv M, Zeng H, He Y, Zhang J, Tan G. Dexmedetomidine promotes liver regeneration in mice after $70 \%$ partial hepatectomy by suppressing NLRP3 inflammasome not TLR4/NFKB. Int. Immunopharmacol. 54, 46-51 (2018).

27. Brenner C, Galluzzi L, Kepp O, Kroemer G. Decoding cell death signals in liver inflammation. J. Hepatol. 59(3), 583-594 (2013).

28. Emadali A, Muscatelli-Groux B, Delom F et al. Proteomic analysis of ischemia-reperfusion injury upon human liver transplantation reveals the protective role of IQGAP1. Mol. Cell. Proteomics 5(7), 1300-1313 (2006).

29. Emadali A, Metrakos PP, Kalantari F et al. Proteomic analysis of tyrosine phosphorylation during human liver transplantation. Proteome Sci. 5, 1 (2007). 
30. Vascotto C, Cesaratto L, D’Ambrosio C et al. Proteomic analysis of liver tissues subjected to early ischemia/reperfusion injury during human orthotopic liver transplantation. Proteomics 6, 3455-3465 (2006).

31. Inoue $\mathrm{T}$, Abe $\mathrm{C}$, Sung SS et al. Vagus nerve stimulation mediates protection from kidney ischemia-reperfusion injury through alpha7nAChR+ splenocytes. J. Clin. Invest. 126, 1939-1952 (2016). 